\title{
Existence and Uniqueness of the Solutions for Fractional Differential Equations with Nonlinear Boundary Conditions
}

\author{
Xiping Liu, Fanfan Li, Mei Jia, and Ertao Zhi \\ College of Science, University of Shanghai for Science and Technology, Shanghai 200093, China \\ Correspondence should be addressed to Xiping Liu; xipingliu@163.com
}

Received 3 March 2014; Accepted 29 May 2014; Published 19 June 2014

Academic Editor: Abdelghani Bellouquid

Copyright ( 2014 Xiping Liu et al. This is an open access article distributed under the Creative Commons Attribution License, which permits unrestricted use, distribution, and reproduction in any medium, provided the original work is properly cited.

\begin{abstract}
We study the existence and uniqueness of the solutions for the boundary value problem of fractional differential equations with nonlinear boundary conditions. By using the upper and lower solutions method in reverse order and monotone iterative techniques, we obtain the sufficient conditions of both the existence of the maximal and minimal solutions between an upper solution and a lower solution and the uniqueness of the solutions for the boundary value problem and present the iterative sequence for calculating the approximate analytical solutions of the boundary value problem and the error estimate. An example is also given to illustrate the main results.
\end{abstract}

\section{Introduction}

In this paper, we study the following fractional differential equation with nonlinear boundary conditions:

$$
\begin{gathered}
{ }^{C} D_{0+}^{\delta} u(t)-M u(t)=f(t, u(t)), \quad t \in J, \\
u^{\prime}(0)=0, \quad u(0)=g(u(T)),
\end{gathered}
$$

where $J=[0, T], 0<T<+\infty . M \geq 0$ is a constant. $f \in C(J \times$ $\mathbb{R}, \mathbb{R}), g \in C^{1}(\mathbb{R}, \mathbb{R})$ are given functions. ${ }^{C} D_{0+}^{\delta}$ is the standard Caputo fractional derivative of order $\delta$ with $1<\delta<2$.

With the development of mathematics, fractional derivative occurs more and more frequently in different research areas, such as physics, mechanics, electricity, and economics (see $[1,2])$. At the same time, significant progress has also been made on the studies of fractional differential equations (see [3-12]).

The upper and lower solutions method and monotone iterative techniques have been widely used in the studies for the boundary value problems of integer-order differential equations (see [13-15] and the references therein). In [13], the authors studied first order differential equation

$$
\begin{gathered}
u^{\prime}(t)=f(t, u(t), u(\theta(t))), \\
g(u(0))=u(T) .
\end{gathered}
$$

By using the upper and lower solutions method and the monotone iterative techniques, the authors concluded that (2) exists the maximal and minimal solutions in $[\beta(t), \alpha(t)]$, where $\alpha(t), \beta(t)$ are the lower and upper solutions of (2).

As for the fractional differential problems, upper and lower solutions and monotone iterative techniques have also been widely used in the studies of boundary value problem (see [16-18]). For example, in [16], by using the method, the authors discussed the existence and uniqueness of solutions for the fractional differential equation with linear boundary condition

$$
\begin{aligned}
D^{\delta} u(t)-M u(t) & =f(t, u(t)), \quad t \in J, 0<\delta<1, \\
u(0)=r u(T), &
\end{aligned}
$$

where $M \geq 0,0<r<1 / E_{\delta, 1}\left(M T^{\delta}\right)$.

In this paper, we study the existence and uniqueness of the solutions for the boundary value problem of the fractional differential equation (1), which has nonlinear 
boundary conditions. It is very difficult to get the iterative sequence which is used to obtain the solutions since the boundary value problem has nonlinear boundary conditions. By using the upper and lower solutions method in reverse order and monotone iterative techniques, we not only obtain the sufficient conditions that the boundary value problem (1) has the maximal and minimal solutions between an upper solution and a lower solution as well as having the unique solution, but also present the iterative sequence for calculating the approximate analytical solutions of the boundary value problem and the error estimate. An example is also given to illustrate the main results.

The organization of this paper is as follows. In Section 2, we provide the necessary background and comparison principles which are used to prove our main results. In Section 3, we consider a linear problem associated with (1). In Section 4, by using the method of upper and lower solutions and the monotone iterative techniques, we obtain the existence and uniqueness solutions of (1). In Section 5, the calculation method of the approximate analytical solutions for the boundary value problems (1) is obtained. In Section 6, an example is presented to illustrate our main results.

\section{Preliminaries and Comparison Principles}

In this section, we present some basic definitions, lemmas, and comparison principles which play important roles in our investigation.

Definition 1 (See $[3,4]$ ). Let $\delta>0$ and function $u$ : $(0,+\infty) \rightarrow \mathbb{R}$. The Riemann-Liouville fractional integral operator of order $\delta$ of $u$ is defined by

$$
I^{\delta} u(t)=\frac{1}{\Gamma(\delta)} \int_{0}^{t}(t-s)^{\delta-1} u(s) \mathrm{d} s
$$

provided the integral exists.

The Caputo derivative of order $\delta$ for function $u$ : $(0,+\infty) \rightarrow \mathbb{R}$ is given by

$$
{ }^{C} D^{\delta} u(t)=\frac{1}{\Gamma(n-\alpha)} \int_{0}^{t} \frac{u^{(n)}(s)}{(t-s)^{\delta+1-n}} \mathrm{~d} s
$$

provided the right side is pointwise defined on $(0,+\infty)$, where $n \in \mathbb{N}^{+}$with $n-1<\delta<n$.

$$
\text { If } \delta=n \in \mathbb{N}^{+} \text {, then }{ }^{C} D^{\delta} u(t)=u^{(n)}(t) \text {. }
$$

Definition 2. Let $A C[0,1]$ be the space of the functions $u$ which are absolutely continuous on $[0,1]$. We denote $A C^{n}[0,1]$ by the set of the functions $u$ which have continuous derivatives up to order $n-1$ on $[0,1]$ such that $u^{(n-1)} \in$ $A C[0,1]$. In particular, $A C^{1}[0,1]=A C[0,1]$.

Lemma 3 (see [3]). If $u \in A C^{n}[0,1]$, then the Caputo fractional derivative ${ }^{C} D^{\delta} u(t)$ exists almost everywhere on $[0,1]$, where $n$ is the smallest integer greater than or equal to $\delta$.
Lemma 4 (see [3]). Suppose $\delta>0$ and $u \in A C^{n}(J)$. Then

$$
\begin{gathered}
I^{\delta C} D^{\delta} u(t)=u(t)+c_{0}+c_{1} t+c_{2} t^{2}+\cdots+c_{n-1} t^{n-1}, \\
c_{k}=\frac{u^{(k)}(0)}{k !}, \quad k=0,1,2, \ldots, n-1,
\end{gathered}
$$

where $n$ is the smallest integer greater than or equal to $\delta$.

Definition 5. One says $u$ is a solution of the boundary value problem (1) if $u \in A C^{2}(J)$ and ${ }^{C} D^{\delta} u \in C(J)$ and satisfies (1).

Definition 6 (see [4]). Let the real $n>0$. The function $E_{n}$ is defined by

$$
E_{n}(z)=\sum_{j=0}^{\infty} \frac{z^{j}}{\Gamma(j n+1)},
$$

whenever the series converges called the Mittag-Leffler function of order $n$.

Definition 7 (see [4]). Let $n_{1}, n_{2}>0$. The function $E_{n_{1}, n_{2}}$ is defined by

$$
E_{n_{1}, n_{2}}(z)=\sum_{j=0}^{\infty} \frac{z^{j}}{\Gamma\left(j n_{1}+n_{2}\right)},
$$

whenever the series converges called the two-parameter Mittag-Leffler function with parameters $n_{1}$ and $n_{2}$.

Remark 8 (see [4]). It is evident that the one-parameter Mittag-Leffler functions may be defined in terms of their twoparameter counterparts via the relation $E_{n}(z)=E_{n, 1}(z)$.

Lemma 9 (see [4], Theorem 4.1). The two-parameter MittagLeffler function $E_{n_{1}, n_{2}}(z)$ for some $n_{1}, n_{2}>0$. The power series defining $E_{n_{1}, n_{2}}(z)$ is convergent for all $z \in \mathbb{R}$.

Lemma 10 (see [3], Theorem 4.3). Let $h \in C(J)$. The Cauchy problem

$$
\begin{gathered}
{ }^{C} D_{0+}^{\delta} u(t)-M u(t)=h(t), \quad t \in J, \\
u(0)=b, \quad u^{\prime}(0)=d, \quad b, d \in \mathbb{R},
\end{gathered}
$$

with $1<\delta<2$ and $M \in \mathbb{R}$ has a unique solution

$$
\begin{aligned}
u(t)= & b E_{\delta}\left(M t^{\delta}\right)+d E_{\delta, 2}\left(M t^{\delta}\right) t \\
& +\int_{0}^{t}(t-s)^{\delta-1} E_{\delta, \delta}\left(M(t-s)^{\delta}\right) h(s) \mathrm{d} s .
\end{aligned}
$$

Lemma 11 (see [19]). Let $E$ be a partially ordered Banach space, $x_{n} \leq y_{n}(n=1,2,3, \ldots)$, if $x_{n} \rightarrow x^{*}, y_{n} \rightarrow y_{0}^{*}$. Then $x^{*} \leq y^{*}$.

In this paper, we assume the following conditions are satisfied.

(H0) A given function $c \in A C^{2}(J)$ and ${ }^{C} D_{0+}^{\delta} c \in C(J) . c$ is monotone decreasing on $J$, and $c(0)=c^{\prime}(0)=0$, $c(T) \leq-1$.

(H1) $g \in C^{1}(\mathbb{R}, \mathbb{R}), g(0) \geq 0$, and $0<g^{\prime}(x)<1 / E_{\delta}\left(M T^{\delta}\right)$. 
Definition 12. Let $\alpha \in A C^{2}(J)$ and ${ }^{C} D_{0+}^{\delta} \alpha \in C(J)$; we say that $\alpha$ is a lower solution of the boundary value problem (1), if

$$
\begin{gathered}
{ }^{C} D_{0+}^{\delta} \alpha(t)-M \alpha(t) \geq f(t, \alpha(t))-a_{\alpha}(t), \quad t \in J, \\
\alpha^{\prime}(0) \geq 0,
\end{gathered}
$$

where

$$
\begin{aligned}
& a_{\alpha}(t) \\
& \quad= \begin{cases}0, & g(\alpha(T)) \leq \alpha(0), \\
\left(\begin{array}{l}
\left.{ }^{C} D_{0+}^{\delta} c(t)-M c(t)\right) \\
\times\left(\alpha(T)-g^{-1}(\alpha(0))\right),
\end{array}\right. & g(\alpha(T))>\alpha(0) .\end{cases}
\end{aligned}
$$

Let $\beta \in A C^{2}(J)$ and ${ }^{C} D_{0+}^{\delta} \beta \in C(J)$; we say that $\beta$ is an upper solution of the boundary value problem (1), if

$$
\begin{gathered}
{ }^{C} D_{0+}^{\delta} \beta(t)-M \beta(t) \leq f(t, \beta(t))+b_{\beta}(t), \quad t \in J, \\
\beta^{\prime}(0) \leq 0,
\end{gathered}
$$

where

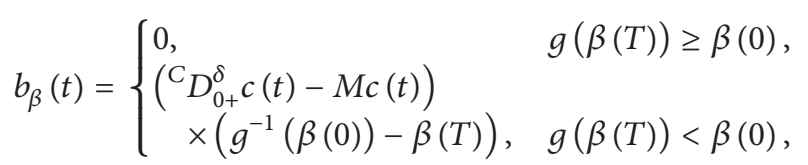

and $c(t)$ is defined in $(\mathrm{H} 0)$.

Lemma 13. Let $g_{1} \in C^{1}(\mathbb{R}, \mathbb{R})$ be any function with $g_{1}(0) \geq 0$ and $0<g_{1}^{\prime}(u)<1 / E_{\delta}\left(M T^{\delta}\right)$. If $u \in A C^{2}(J)$ and ${ }^{C} D_{0+}^{\delta} u \in$ $C(J)$ and satisfies

$$
\begin{gathered}
{ }^{C} D_{0+}^{\delta} u(t)-M u(t) \geq 0, \quad t \in J, \\
u^{\prime}(0) \geq 0, \quad u(0) \geq g_{1}(u(T)),
\end{gathered}
$$

then $u(t) \geq 0$ for $t \in J$.

Proof. If $u(0)<0$, by the boundary conditions, we have

$$
0>u(0) \geq g_{1}(u(T))=g_{1}(0)+g_{1}^{\prime}(\xi) u(T) \geq g_{1}^{\prime}(\xi) u(T),
$$

where $\xi$ is a constant between 0 and $u(T)$.

Because $g_{1}(0) \geq 0$ and $0<g_{1}^{\prime}(u)<1 / E_{\delta}\left(M T^{\delta}\right)$, then $u(T)<0$ and $u(0)>\left(1 / E_{\delta}\left(M T^{\delta}\right)\right) u(T)$; that is,

$$
u(T)<u(0) E_{\delta}\left(M T^{\delta}\right)
$$

Let $h(t)={ }^{C} D_{0+}^{\delta} u(t)-M u(t), u(0)=b$, and $u^{\prime}(0)=d$, and then $h(t) \geq 0$ for $t \in J$ and $d \geq 0$.

By Lemma 10, we can get that the Cauchy problem

$$
\begin{gathered}
{ }^{C} D_{0+}^{\delta} u(t)-M u(t)=h(t), \quad t \in J, \\
u(0)=b, \quad u^{\prime}(0)=d,
\end{gathered}
$$

has a unique solution

$$
\begin{aligned}
u(t)=b E_{\delta} & \left(M t^{\delta}\right)+d E_{\delta, 2}\left(M t^{\delta}\right) t \\
& +\int_{0}^{t}(t-s)^{\delta-1} E_{\delta, \delta}\left(M(t-s)^{\delta}\right) h(s) \mathrm{d} s .
\end{aligned}
$$

So we can obtain that

$$
\begin{aligned}
u(T)= & b E_{\delta}\left(M T^{\delta}\right)+d T E_{\delta, 2}\left(M T^{\delta}\right) \\
& +\int_{0}^{T}(T-s)^{\delta-1} E_{\delta, \delta}\left(M(T-s)^{\delta}\right) h(s) \mathrm{d} s \\
\geq & b E_{\delta}\left(M T^{\delta}\right)=u(0) E_{\delta}\left(M T^{\delta}\right),
\end{aligned}
$$

which is contradictory to (17).

Hence, $b=u(0) \geq 0$. By (19), we can obtain that $u(t) \geq 0$ for $t \in J$.

Let the function $g_{1}(x)=r_{1} x$ in Lemma 13; we can obtain Corollary 14.

Corollary 14. If there exists a constant $r_{1}$ with $0<r_{1}<$ $1 / E_{\delta}\left(M T^{\delta}\right)$ such that $u \in A C^{2}(J)$ and ${ }^{C} D_{0+}^{\delta} u \in C(J)$ and satisfies

$$
\begin{aligned}
& { }^{C} D_{0+}^{\delta} u(t) \geq M u(t), \quad t \in J, \\
& u^{\prime}(0) \geq 0, \quad u(0) \geq r_{1} u(T),
\end{aligned}
$$

then $u(t) \geq 0$ on $J$.

Corollary 15. Suppose (HO) holds, and there exists a constant $r_{2}>E_{\delta}\left(M T^{\delta}\right)$ such that $u \in A C^{2}(J)$ and ${ }^{C} D_{0+}^{\delta} u \in C(J)$ and satisfies

$$
\begin{aligned}
&{ }^{C} D_{0+}^{\delta} u(t) \geq M u(t)-\left({ }^{C} D_{0+}^{\delta} c(t)-M c(t)\right) \\
& \times\left(u(T)-r_{2} u(0)\right), \quad t \in J, \\
& u^{\prime}(0) \geq 0, \quad u(T) \geq r_{2} u(0)
\end{aligned}
$$

then $u(t) \geq 0$ for $t \in J$.

Proof. Let $g_{1}(x)=\left(1 / r_{2}\right) x$ and $y(t)=u(t)+c(t)(u(T)-$ $\left.r_{2} u(0)\right), t \in J$.

Since $c(t)$ is monotone decreasing and $c(0)=0$, then $c(t) \leq 0$ on $J$. So $y(t) \leq u(t), t \in J$.

By (22), we have

$$
\begin{aligned}
{ }^{C} D_{0+}^{\delta} y(t)-M y(t) \\
={ }^{C} D_{0+}^{\delta} u(t)-M u(t) \\
\quad+\left({ }^{C} D_{0+}^{\delta} c(t)-M c(t)\right)\left(u(T)-r_{2} u(0)\right) \geq 0,
\end{aligned}
$$

and $y^{\prime}(0)=u^{\prime}(0) \geq 0$.

Because $c(0)=0$ and $c(T) \leq-1$, then $y(T)=u(T)+$ $c(T)\left(u(T)-r_{2} u(0)\right) \leq r_{2} u(0)=r_{2} y(0)$.

So $y(0) \geq\left(1 / r_{2}\right) y(T)=g_{1}(u(T))$. 
By Lemma 13, we can get that $y(t) \geq 0$, which implies that $u(t) \geq 0$ for $t \in J$.

\section{Boundary Value Problems for the Linear Equation}

In this section, we consider the existence and uniqueness of solutions for the linear fractional differential equation with nonlinear boundary conditions

$$
\begin{gathered}
{ }^{C} D_{0+}^{\delta} u(t)-M u(t)=h(t), \quad t \in J, \\
u^{\prime}(0)=0, \quad u(0)=g(u(T)),
\end{gathered}
$$

where $h \in C(J)$.

Theorem 16. Assume that (HO) and (H1) hold; there exist a lower solution $\alpha$ and an upper solution $\beta$ of the boundary value problem (24) with $\alpha(t) \geq \beta(t)$ on J. Then the boundary value problem (24) has a unique solution $u$. Moreover, $\alpha \geq u \geq \beta$; that is, $\alpha(t) \geq u(t) \geq \beta(t)$ for $t \in J$.

Proof. (1) We show that the solution of (24) is unique if it exists.

Suppose that $u_{1}, u_{2}$ are two solutions of (24) and let $v=$ $u_{1}-u_{2}$. Then,

$$
\begin{gathered}
{ }^{C} D_{0+}^{\delta} v(t)-M v(t)=0, \quad t \in J, \\
v^{\prime}(0)=0, \\
v(0)=g\left(u_{1}(T)\right)-g\left(u_{2}(T)\right)=g^{\prime}\left(\tau_{1}\right) v(T),
\end{gathered}
$$

where $\tau_{1}$ is a constant between $u_{1}(T)$ and $u_{2}(T)$ and $0<$ $g^{\prime}\left(\tau_{1}\right)<1 / E_{\delta}\left(M T^{\delta}\right)$ is a constant.

By Corollary 14, we have that $v(t) \geq 0$; that is, $u_{1}(t) \geq$ $u_{2}(t)$ for $t \in J$.

Similarly, we can also obtain that $u_{2}(t) \geq u_{1}(t)$, for $t \in J$.

Hence, $u_{1}=u_{2}$.

(2) We prove that $\beta \leq u \leq \alpha$ if $u$ is a solution of the boundary value problem (24).

Let $m=\alpha-u$.

If $g(\alpha(T)) \leq \alpha(0)$, then $a_{\alpha}(t)=0$ on $J$. We have

$$
\begin{gathered}
{ }^{C} D_{0+}^{\delta} m(t)-M m(t) \geq 0, \quad t \in J, \\
m^{\prime}(0) \geq 0, \\
m(0) \geq g(\alpha(T))-g(u(T))=g^{\prime}\left(\tau_{2}\right) m(T),
\end{gathered}
$$

where $\tau_{2}$ is a constant between $u(T)$ and $\alpha(T)$.

We denote $r_{1}=g^{\prime}\left(\tau_{2}\right)$. Hence, $0<r_{1}<1 / E_{\delta}\left(M T^{\delta}\right)$.

By Corollary 14, we can obtain that $m(t)=\alpha(t)-u(t) \geq 0$ on $J$.
If $g(\alpha(T))>\alpha(0)$, then $a_{\alpha}(t)=\left({ }^{C} D_{0+}^{\delta} c(t)-M c(t)\right)(\alpha(T)-$ $\left.g^{-1}(\alpha(0))\right)$. We have that

$$
\begin{aligned}
&{ }^{C} D_{0+}^{\delta} m(t)-M m(t) \\
&={ }^{C} D_{0+}^{\delta} \alpha(t)-M \alpha(t)-{ }^{C} D_{0+}^{\delta} u(t)+M u(t) \\
& \geq-\left({ }^{C} D_{0+}^{\delta} c(t)-M c(t)\right)\left(\alpha(T)-g^{-1}(\alpha(0))\right) \\
&=-\left({ }^{C} D_{0+}^{\delta} c(t)-M c(t)\right) \\
& \times\left(\alpha(T)-u(T)+g^{-1}(u(0))-g^{-1}(\alpha(0))\right) \\
&=-\left({ }^{C} D_{0+}^{\delta} c(t)-M c(t)\right)\left(m(T)-\left(g^{-1}\right)^{\prime}\left(\tau_{3}\right) m(0)\right),
\end{aligned}
$$

where $\tau_{3}$ is a constant between $\alpha(0)$ and $u(0)$.

We denote $r_{2}=\left(g^{-1}\right)^{\prime}\left(\tau_{3}\right)$. Hence, $r_{2}>E_{\delta}\left(M T^{\delta}\right)$.

We can show $m^{\prime}(0) \geq 0, m(T) \geq g^{-1}(\alpha(0))-g^{-1}(u(0))=$ $r_{2} m(0)$.

By Corollary 15 , we have $m(t) \geq 0$; that is, $\alpha(t) \geq u(t)$ on $J$. So $\alpha(t) \geq u(t)$ on $J$.

Similarly, we can obtain that $u(t) \geq \beta(t)$ for $J$. Therefore, $\beta \leq u \leq \alpha$.

(3) We prove that the problem (24) has a unique solution. Let

$$
\begin{aligned}
& p(t)= \begin{cases}\alpha(t), & g(\alpha(T)) \leq \alpha(0), \\
\alpha(t)+c(t) & \\
\quad \times\left(\alpha(T)-g^{-1}(\alpha(0))\right), & g(\alpha(T))>\alpha(0),\end{cases} \\
& q(t)= \begin{cases}\beta(t), & g(\beta(T)) \geq \beta(0), \\
\beta(t)-c(t) & \\
\times\left(g^{-1}(\beta(0))-\beta(T)\right), & g(\beta(T))<\beta(0) .\end{cases}
\end{aligned}
$$

It is obvious that $p, q \in A C^{2}(J),{ }^{C} D_{0+}^{\delta} p,{ }^{C} D_{0+}^{\delta} q \in C(J)$, and $p(0)=\alpha(0), q(0)=\beta(0)$. If $g(\alpha(T)) \leq \alpha(0)$, we have $p(T)=\alpha(T)$. If $g(\alpha(T))>\alpha(0)$, we have $p(T) \leq g^{-1}(\alpha(0))$; that is,

$$
g(p(T)) \leq p(0)
$$

Similarly, we can get

$$
g(q(T)) \geq q(0)
$$

If $g(\alpha(T)) \leq \alpha(0)$, we have

$$
{ }^{C} D_{0+}^{\delta} p(t)-M p(t) \geq h(t), \quad t \in J .
$$


If $g(\alpha(T))>\alpha(0)$, since $\alpha$ is a lower solution of boundary value problem (24), then ${ }^{C} D_{0+}^{\delta} \alpha(t)-M \alpha(t) \geq h(t)-a_{\alpha}(t)$. And by (12), we can obtain that

$$
\begin{aligned}
&{ }^{C} D_{0+}^{\delta} p(t)-M p(t) \\
&=\left({ }^{C} D_{0+}^{\delta} \alpha(t)-M \alpha(t)\right) \\
& \quad+\left({ }^{C} D_{0+}^{\delta} c(t)-M c(t)\right)\left(\alpha(T)-g^{-1}(\alpha(0))\right) \\
& \geq h(t)-a_{\alpha}(t) \\
&+\left({ }^{C} D_{0+}^{\delta} c(t)-M c(t)\right)\left(\alpha(T)-g^{-1}(\alpha(0))\right) \\
&= h(t), \quad t \in J .
\end{aligned}
$$

Hence, we obtain

$$
{ }^{C} D_{0+}^{\delta} p(t)-M p(t) \geq h(t), \quad t \in J .
$$

Using the same way as mentioned above, we can get that

$$
{ }^{C} D_{0+}^{\delta} q(t)-M q(t) \leq h(t), \quad t \in J .
$$

Since (H0) holds, it is easy to see that $c(t) \leq 0$ for $t \in J$. We can easily get that $\beta(t) \leq q(t)$ and $p(t) \leq \alpha(t)$ for $t \in J$.

Let $n(t)=p(t)-q(t)$. It follows from (34) and (35) that

$$
\begin{aligned}
& { }^{C} D_{0+}^{\delta} n(t)-M n(t) \\
& ={ }^{C} D_{0+}^{\delta} p(t)-M p(t)-{ }^{C} D_{0+}^{\delta} q(t)+M q(t) \geq 0, \\
& n^{\prime}(0)=p^{\prime}(0)-q^{\prime}(0)=\alpha^{\prime}(0)-\beta^{\prime}(0) \geq 0, \\
& n(0)=p(0)-q(0) \geq g(p(T))-g(q(T)) \\
& \quad=g^{\prime}\left(\tau_{4}\right) n(T),
\end{aligned}
$$

where $\tau_{4}$ is a constant between $p(T)$ and $q(T)$ and $0<$ $g^{\prime}\left(\tau_{4}\right)<1 / E_{\delta}\left(M T^{\delta}\right)$ is a constant.

By Corollary 14, we have $n(t) \geq 0$ for $t \in J$; that is,

$$
p(t) \geq q(t) \quad \text { for } t \in J .
$$

According to Lemma 10, for each $\lambda \in \mathbb{R}$, the Cauchy problem

$$
\begin{gathered}
{ }^{C} D_{0+}^{\delta} u(t)-M u(t)=h(t), \\
u^{\prime}(0)=0, \quad u(0)=\lambda,
\end{gathered}
$$

has a unique solution

$$
\begin{aligned}
u(t, \lambda)= & \lambda E_{\delta}\left(M t^{\delta}\right) \\
& +\int_{0}^{t}(t-s)^{\delta-1} E_{\delta, \delta}\left(M(t-s)^{\delta}\right) h(s) \mathrm{d} s, \quad t \in J .
\end{aligned}
$$

In the following, we show that $q(T) \leq u(T, \lambda) \leq p(T)$ for each $\lambda \in[g(q(T)), g(p(T))]$, where $u(t, \lambda)$ is defined by (39).
If $u(T, \lambda)<q(T)$, we denote $l(t)=u(t, \lambda)-q(t)$, and then

$$
\begin{gathered}
l(T)<0, \\
l(0)=u(0, \lambda)-q(0) \geq \lambda-g(q(T)) \geq 0 .
\end{gathered}
$$

So $l(0) \geq r_{1} l(T)$, for any $r_{1} \in\left(0,1 / E_{\delta}\left(M T^{\delta}\right)\right)$.

It follows that $c^{\prime}(0)=0$ from (H0), and by (29), we have that $q^{\prime}(0)=\beta^{\prime}(0)$. Then

$$
l^{\prime}(0)=u^{\prime}(0, \lambda)-q^{\prime}(0)=-\beta^{\prime}(0) \geq 0 .
$$

And from (35), we have

$$
\begin{aligned}
{ }^{C} D_{0+}^{\delta} & l(t)-M l(t) \\
& ={ }^{C} D_{0+}^{\delta} u(t)-M u(t)-\left({ }^{C} D_{0+}^{\delta} q(t)-M q(t)\right) \\
& =h(t)-\left({ }^{C} D_{0+}^{\delta} q(t)-M q(t)\right) \\
& \geq 0 .
\end{aligned}
$$

By Corollary 14, we can obtain that $l(t) \geq 0$, for all $t \in J$, which is a contradiction to (40). So

$$
u(T, \lambda) \geq q(T)
$$

Similarly, we can get

$$
u(T, \lambda) \leq p(T)
$$

Let $H(\lambda)=g(u(T, \lambda))-\lambda$. Since $H(g(q(T))) H(g(p(T))) \leq 0$ and

$$
\begin{aligned}
H^{\prime}(\lambda) & =g^{\prime}(u(T, \lambda)) u_{\lambda}^{\prime}(T, \lambda)-1 \\
& <\frac{1}{E_{\delta}\left(M T^{\delta}\right)} \cdot E_{\delta}\left(M T^{\delta}\right)-1=0,
\end{aligned}
$$

then $H(\lambda)$ is strictly monotone decreasing and continuous for $\lambda \in[g(q(T)), g(p(T))]$.

Hence, we can get that $H(\lambda)=0$ has a unique solution $\lambda_{0} \in[g(q(T)), g(p(T))]$ with $g\left(u\left(T, \lambda_{0}\right)\right)=\lambda_{0}=u(0)$.

It is easy to see that $u\left(t, \lambda_{0}\right)$ is the unique solution of the boundary value problem (24).

\section{Existence and Uniqueness of the Solutions for Boundary Value Problem}

In this section, we study the existence and uniqueness of the solutions for fractional differential equation with nonlinear boundary conditions (1).

Let $E=C(J)$ endowed with norm $\|u\|=\max _{t \in J}|u(t)|$ for $u \in E$. Then $E$ is a Banach space. Denote

$$
[\beta, \alpha]=\{u \in C(J): \beta(t) \leq u(t) \leq \alpha(t), t \in J\},
$$

and $\beta \leq \alpha$ if and only if $\beta(t) \leq \alpha(t)$ on $J$. 
Theorem 17. Suppose that (HO) and (H1) hold; there exist $\alpha_{0}$, $\beta_{0}$ which are lower and upper solutions of the boundary value problem (1) with $\beta_{0}(t) \leq \alpha_{0}(t)$ for $t \in J$. And $f$ satisfies

(H2) $f\left(t, x_{2}\right)-f\left(t, x_{1}\right) \geq 0$ for any $\beta_{0} \leq x_{1} \leq x_{2} \leq \alpha_{0}$, $t \in J$.

Then there exist monotone sequences $\left\{\alpha_{n}\right\},\left\{\beta_{n}\right\}$ such that

$$
\lim _{n \rightarrow \infty} \alpha_{n}(t)=\alpha^{*}(t), \quad \lim _{n \rightarrow \infty} \beta_{n}(t)=\beta^{*}(t),
$$

converging uniformly on $J$, and $\beta^{*}, \alpha^{*}$ are the minimal and the maximal solutions of (1) on $\left[\beta_{0}, \alpha_{0}\right]$.

Proof. We denote $D=\left[\beta_{0}, \alpha_{0}\right]$. For any $\gamma \in D$, we consider the following boundary value problem:

$$
\begin{gathered}
{ }^{C} D_{0+}^{\delta} u(t)-M u(t)=f(t, \gamma(t)), \quad t \in J, \\
u^{\prime}(0)=0, \quad u(0)=g(u(T)) .
\end{gathered}
$$

Since $\alpha_{0}(t), \beta_{0}(t)$ are lower and upper solutions of the boundary value problem (1), by (H2), we have

$$
\begin{aligned}
{ }^{C} D_{0+}^{\delta} \alpha_{0}(t)-M \alpha_{0}(t) & \geq f\left(t, \alpha_{0}(t)\right)-a_{\alpha}(t) \\
& \geq f(t, \gamma(t))-a_{\alpha}(t), \\
{ }^{C} D_{0+}^{\delta} \beta_{0}(t)-M \beta_{0}(t) & \leq f\left(t, \beta_{0}(t)\right)-b_{\beta}(t) \\
& \leq f(t, \gamma(t))-b_{\beta}(t),
\end{aligned}
$$

for $t \in J$.

Therefore, $\alpha_{0}(t), \beta_{0}(t)$ are also the lower and upper solutions of the boundary value problem (49), respectively.

In view of Theorem 16, the boundary value problem (49) has a unique solution $u$ and $u \in D$.

Define $P:\left[\beta_{0}, \alpha_{0}\right] \rightarrow\left[\beta_{0}, \alpha_{0}\right]$ by $P \gamma=u$. Hence, $\alpha_{0} \geq$ $P \alpha_{0}, \beta_{0} \leq P \beta_{0}$.

We will show that $P x_{1} \leq P x_{2}$ if $\beta_{0} \leq x_{1} \leq x_{2} \leq \alpha_{0}$.

Let $m=P x_{2}-P x_{1}$. By (H2) and (49), we have

$$
\begin{gathered}
{ }^{C} D_{0+}^{\delta} m(t)-M m(t)=f\left(t, x_{2}(t)\right)-f\left(t, x_{1}(t)\right) \geq 0, \\
m^{\prime}(0)=0, \quad m(0)=g^{\prime}\left(\tau_{5}\right) m(T),
\end{gathered}
$$

where $\tau_{5}$ is a constant between $P x_{1}(T)$ and $P x_{2}(T)$, and denote $r_{1}=g^{\prime}\left(\tau_{5}\right)<1 / E_{\delta, 1}\left(M T^{\delta}\right)$. By Corollary $14, m(t) \geq 0$, which implies $P x_{1} \leq P x_{2}$. Hence, $P$ is monotone increasing in $\left[\beta_{0}, \alpha_{0}\right]$.

Let $\alpha_{n}=P \alpha_{n-1}$ and $\beta_{n}=P \beta_{n-1}$ for $n=1,2, \ldots$. We can get monotone iterative sequences

$$
\beta_{1} \leq \beta_{2} \leq \cdots \leq \beta_{n} \leq \cdots \leq \alpha_{n} \leq \cdots \leq \alpha_{2} \leq \alpha_{1} .
$$

Therefore, there exist $\alpha^{*}, \beta^{*} \in E$, such that

$$
\lim _{n \rightarrow \infty} \alpha_{n}(t)=\alpha^{*}(t), \quad \lim _{n \rightarrow \infty} \beta_{n}(t)=\beta^{*}(t) .
$$

It is easy to see that $\alpha_{n}(t)$ satisfies

$$
\begin{gathered}
{ }^{C} D_{0+}^{\delta} \alpha_{n}(t)-M \alpha_{n}(t)=f\left(t, \alpha_{n-1}(t)\right), \\
\alpha_{n}^{\prime}(0)=0, \quad \alpha_{n}(0)=g\left(\alpha_{n}(T)\right) .
\end{gathered}
$$

By Lemma 10, we have

$$
\begin{aligned}
\alpha_{n}(t)= & \alpha_{n}(0) E_{\delta}\left(M t^{\delta}\right) \\
& +\int_{0}^{t}(t-s)^{\delta-1} E_{\delta, \delta}\left(M(t-s)^{\delta}\right) f\left(s, \alpha_{n-1}(s)\right) \mathrm{d} s .
\end{aligned}
$$

Because $f$ is continuous and $\alpha_{n-1} \in D$, there exists a constant $L>0$ such that

$$
\begin{gathered}
\left|f\left(t, \alpha_{n-1}(t)\right)\right| \leq L, \quad t \in J, n=1,2, \ldots, \\
f\left(t, \alpha_{n-1}(t)\right) \longrightarrow f\left(t, \alpha^{*}(t)\right), \quad t \in J, n \longrightarrow \infty .
\end{gathered}
$$

By Lebesgue dominated convergence theorem, we can get that

$$
\begin{aligned}
\lim _{n \rightarrow \infty} \alpha_{n}(t)= & \lim _{n \rightarrow \infty} \alpha_{n}(0) E_{\delta}\left(M t^{\delta}\right) \\
& +\lim _{n \rightarrow \infty} \int_{0}^{t}(t-s)^{\delta-1} f\left(s, \alpha_{n-1}(s)\right) \mathrm{d} s \\
= & \alpha^{*}(0) E_{\delta}\left(M t^{\delta}\right)+\int_{0}^{t}(t-s)^{\delta-1} f\left(s, \alpha^{*}(s)\right) \mathrm{d} s .
\end{aligned}
$$

That is,

$$
\alpha^{*}(t)=\alpha^{*}(0) E_{\delta}\left(M t^{\delta}\right)+\int_{0}^{t}(t-s)^{\delta-1} f\left(s, \alpha^{*}(s)\right) \mathrm{d} s .
$$

Therefore,

$$
\alpha^{*}(t)=P \alpha^{*}(t) \quad \text { for } t \in J
$$

It is similar to show that

$$
\beta^{*}(t)=P \beta^{*}(t) \quad \text { for } t \in J
$$

It is clear that $\alpha^{*}, \beta^{*}$ are solutions of the boundary value problem (1).

Assume $u \in\left[\beta_{0}, \alpha_{0}\right]$ is a solution of the boundary value problem (1). We can easily obtain that $P \beta_{0}(t) \leq P u(t) \leq$ $P \alpha_{0}(t)$ by the fact that $P$ is increasing in $\left[\beta_{0}, \alpha_{0}\right]$. That is, $\beta_{1}(t) \leq u(t) \leq \alpha_{1}(t)$. Doing this repeatedly, we have $\beta_{n}(t) \leq$ $u(t) \leq \alpha_{n}(t)$, for $n=1,2, \ldots$. From Lemma 11, we obtain that $\beta^{*}(t) \leq u(t) \leq \alpha^{*}(t)$, as $n \rightarrow \infty$.

Hence, $\alpha^{*}, \beta^{*}$ are the maximal and the minimal solutions of the boundary value problem (1), respectively.

Theorem 18. Suppose that the conditions (HO) and (H1) hold; there exist $\alpha_{0}, \beta_{0}$ lower and upper solutions of the boundary value problem (1) with $\beta_{0}(t) \leq \alpha_{0}(t)$ for $t \in J$, respectively. If there exists a constant $k$ with $0<k<\delta(1-$ $\left.a E_{\delta}\left(M T^{\delta}\right)\right) / T^{\delta} E_{\delta, \delta}\left(M T^{\delta}\right)$, where $a=\max _{u \in\left[\beta_{0}, \alpha_{0}\right]} g^{\prime}(u)$, and $f$ satisfies

(H3) $f\left(t, x_{2}\right)-f\left(t, x_{1}\right) \leq k\left(x_{2}-x_{1}\right)$ for any $\beta_{0} \leq x_{1} \leq x_{2} \leq$ $\alpha_{0}, t \in J$, 
then the boundary value problem (1) has a unique solution $u^{*}$ on $\left[\beta_{0}, \alpha_{0}\right]$ and for any $u_{0} \in\left[\beta_{0}, \alpha_{0}\right]$, the iterative sequence $u_{n}=P u_{n-1}, n=0,1,2, \ldots$, converging uniformly to $u^{*}$ on $J$ and its error estimate is

$$
\left\|u_{n}-u^{*}\right\| \leq\left(\frac{k T^{\delta} E_{\delta, \delta}\left(M T^{\delta}\right)}{\delta\left(1-a E_{\delta}\left(M T^{\delta}\right)\right)}\right)^{n}\left\|\alpha_{0}-\beta_{0}\right\| .
$$

Proof. It is easy to check that the conditions of Theorem 17 are satisfied. Then the boundary value problem (1) has the maximal solution and the minimal solution which are denoted by $\alpha^{*}, \beta^{*}$, respectively.

For $\beta_{0} \leq \omega_{1} \leq \omega_{2} \leq \alpha_{0}, t \in J$, we have

$$
\begin{aligned}
P \omega_{2}(t)-P \omega_{1}(t) & \\
= & \left.P \omega_{2}(0)-P \omega_{1}(0)\right) E_{\delta}\left(M t^{\delta}\right) \\
+ & \int_{0}^{t}(t-s)^{\delta-1} E_{\delta, \delta}\left(M(t-s)^{\delta}\right) \\
& \times\left(f\left(s, \omega_{2}(s)\right)-f\left(s, \omega_{1}(s)\right)\right) \mathrm{d} s,
\end{aligned}
$$

and from $(\mathrm{H} 3)$,

$$
\begin{aligned}
& P \omega_{2}(0)-P \omega_{1}(0) \\
& =g\left(P \omega_{2}(T)\right)-g\left(P \omega_{1}(T)\right) \\
& =g^{\prime}(\tau)\left(P \omega_{2}(T)-P \omega_{1}(T)\right) \\
& =g^{\prime}(\tau)\left(\left(P \omega_{2}(0)-P \omega_{1}(0)\right) E_{\delta}\left(M T^{\delta}\right)\right. \\
& +\int_{0}^{T}(T-s)^{\delta-1} E_{\delta, \delta}\left(M(T-s)^{\delta}\right) \\
& \left.\times a\left(f\left(s, \omega_{2}(s)\right)-f\left(s, \omega_{1}(s)\right)\right) \mathrm{d} s\right) \\
& \quad\left(P \omega_{2}(0)-P \omega_{1}(0)\right) E_{\delta}\left(M T^{\delta}\right) \\
& +\int_{0}^{T}(T-s)^{\delta-1} E_{\delta, \delta}\left(M(T-s)^{\delta}\right) \\
& \times a\left(f T_{\delta, \delta}^{\delta}\left(M T^{\delta}\right)\right. \\
& \left.\quad(0)-P \omega_{1}(0)\right) E_{\delta}\left(M T^{\delta}\right) \\
& \left.\left.(s))-f\left(s, \omega_{1}(s)\right)\right) \mathrm{d} s\right)
\end{aligned}
$$

where $\tau \in\left[\omega_{1}(T), \omega_{2}(T)\right] \subset\left[\beta_{0}(T), \alpha_{0}(T)\right]$ and $P$ is defined in Theorem 17.
It follows that $1-a E_{\delta}\left(M T^{\delta}\right)>0$ from $0<g^{\prime}(x)<$ $1 / E_{\delta}\left(M T^{\delta}\right)$ for $x \in \mathbb{R}$. Hence,

$$
\begin{aligned}
& 0 \leq P \omega_{2}(0)-P \omega_{1}(0) \\
& \leq \frac{k a T^{\delta} E_{\delta, \delta}\left(M T^{\delta}\right)}{\delta\left(1-a E_{\delta}\left(M T^{\delta}\right)\right)}\left\|\omega_{2}-\omega_{1}\right\|, \\
& 0 \leq P \omega_{2}(t)-P \omega_{1}(t) \\
& \leq \frac{E \delta\left(M T^{\delta}\right) k a T^{\delta} E_{\delta, \delta}\left(M T^{\delta}\right)}{\delta\left(1-a E_{\delta}\left(M T^{\delta}\right)\right)}\left\|\omega_{2}-\omega_{1}\right\| \\
&+\frac{k T^{\delta} E_{\delta, \delta}\left(M T^{\delta}\right)}{\delta}\left\|\omega_{2}-\omega_{1}\right\| .
\end{aligned}
$$

We can easily get

$$
\left\|P \omega_{2}-P \omega_{1}\right\| \leq \frac{k T^{\delta} E_{\delta, \delta}\left(M T^{\delta}\right)}{\delta\left(1-a E_{\delta}\left(M T^{\delta}\right)\right)}\left\|\omega_{2}-\omega_{1}\right\| .
$$

Because $0<k<\delta\left(1-a E_{\delta}\left(M T^{\delta}\right)\right) / T^{\delta} E_{\delta, \delta}\left(M T^{\delta}\right)$, we have

$$
0<\frac{k T^{\delta} E_{\delta, \delta}\left(M T^{\delta}\right)}{\delta\left(1-a E_{\delta}\left(M T^{\delta}\right)\right)}<1
$$

It implies that $P$ is contraction mapping. By using the contraction mapping principle, the $P$ has a unique fixed point.

Therefore, the boundary value problem (1) has a unique solution; that is, $\alpha^{*}=\beta^{*}$, and we denote it by $u^{*}$.

For any $u_{0} \in\left[\beta_{0}, \alpha_{0}\right]$, let the iterative sequence $u_{n}=$ $P u_{n-1}, n=0,1,2, \ldots$. Similar to (65), we can get

$$
\begin{aligned}
\left\|u_{n}-u^{*}\right\| & \leq \frac{k T^{\delta} E_{\delta, \delta}\left(M T^{\delta}\right)}{\delta\left(1-a E_{\delta}\left(M T^{\delta}\right)\right)}\left\|P u_{n-1}-u^{*}\right\| \\
& \leq \cdots \\
& \leq\left(\frac{k T^{\delta} E_{\delta, \delta}\left(M T^{\delta}\right)}{\delta\left(1-a E_{\delta}\left(M T^{\delta}\right)\right)}\right)^{n}\left\|u_{0}-u^{*}\right\| .
\end{aligned}
$$

It follows that $\left\{u_{n}\right\}$ converging uniformly to $u^{*}$ on $J$ from (66).

Since $u_{0}, u^{*} \in\left[\beta_{0}, \alpha_{0}\right]$, we can obtain that the error estimate of $\left\{u_{n}\right\}$ is

$$
\left\|u_{n}-u^{*}\right\| \leq\left(\frac{k T^{\delta} E_{\delta, \delta}\left(M T^{\delta}\right)}{\delta\left(1-a E_{\delta}\left(M T^{\delta}\right)\right)}\right)^{n}\left\|\alpha_{0}-\beta_{0}\right\| .
$$

\section{The Calculation Method of the Approximate Analytical Solutions}

In this section, we give the calculation method of the approximate analytical solutions for the boundary value problem (1). 
Theorem 19. Suppose (HO) and (H1) hold; there exist a lower solution $\alpha_{0}$ and an upper solution $\beta_{0}$ of the boundary value problem (1) with $\beta_{0}(t) \leq \alpha_{0}(t)$ for $t \in J$. And there exists a constant $\kappa$ with $0<\kappa<\delta\left(1-b E_{\delta}\left(M T^{\delta}\right)\right) / T^{\delta} E_{\delta, \delta}\left(M T^{\delta}\right)$, where $b=\sup _{u \in \mathbb{R}} g^{\prime}(u)<1 / E_{\delta}\left(M T^{\delta}\right)$, such that $f$ satisfies

(H4) $0 \leq f\left(t, x_{2}\right)-f\left(t, x_{1}\right) \leq \kappa\left(x_{2}-x_{1}\right)$ for any $x_{1} \leq x_{2}$, $t \in J$.

Then for any initial value $y_{0} \in\left[\beta_{0}, \alpha_{0}\right]$, the sequence $\left\{y_{n}\right\}$ is defined by

$$
\begin{aligned}
y_{n}(t)= & g\left(y_{n-1}(T)\right) E_{\delta}\left(M t^{\delta}\right) \\
& +\int_{0}^{t}(t-s)^{\delta-1} E_{\delta, \delta}\left(M(t-s)^{\delta}\right) f\left(s, y_{n-1}(s)\right) \mathrm{d} s,
\end{aligned}
$$

converging uniformly to the unique solution ${ }^{*}$ of the boundary value problem (1) on the $\left[\beta_{0}, \alpha_{0}\right]$. Furthermore, the error estimate is

$$
\left\|y_{n}-u^{*}\right\| \leq \frac{\varrho_{0}^{n}-\varrho_{1}^{n+1}}{1-\varrho_{1}}\left\|\alpha_{0}-\beta_{0}\right\|
$$

where

$$
\begin{aligned}
& \varrho_{0}=b E_{\delta}\left(M T^{\delta}\right)+\frac{\kappa T^{\delta} E_{\delta, \delta}\left(M T^{\delta}\right)}{\delta} \\
& \varrho_{1}=\frac{\kappa T^{\delta} E_{\delta, \delta}\left(M T^{\delta}\right)}{\delta\left(1-b E_{\delta}\left(M T^{\delta}\right)\right)} .
\end{aligned}
$$

Proof. Because $0<\kappa<\delta\left(1-b E_{\delta}\left(M T^{\delta}\right)\right) / T^{\delta} E_{\delta, \delta}\left(M T^{\delta}\right)$, where $b=\sup _{u \in \mathbb{R}} g^{\prime}(u)<1 / E_{\delta}\left(M T^{\delta}\right)$, then $b \geq a$ and $\kappa<\delta\left(1-a E_{\delta}\left(M T^{\delta}\right)\right) / T^{\delta} E_{\delta, \delta}\left(M T^{\delta}\right)$, where $a$ is defined by Theorem 18.

Hence, the conditions of Theorem 18 hold, and we can get that the boundary value problem (1) has a unique solution $u^{*}$ on $\left[\beta_{0}, \alpha_{0}\right]$ and for any $u_{0} \in\left[\beta_{0}, \alpha_{0}\right]$, the iterative sequence $u_{n}=P u_{n-1}, n=1,2, \ldots$, converging uniformly to $u^{*}$ on $J$. That is,

$$
\left\|u_{n}-u^{*}\right\| \longrightarrow 0, \quad \text { as } n \longrightarrow \infty \text {. }
$$

By Lemma 10 and the definition of operator $P$, we can easily obtain that $u_{n}=P u_{n-1}$ is equivalent to

$$
\begin{aligned}
u_{n}(t)= & u_{n}(0) E_{\delta}\left(M t^{\delta}\right) \\
& +\int_{0}^{t}(t-s)^{\delta-1} E_{\delta, \delta}\left(M(t-s)^{\delta}\right) f\left(s, u_{n-1}(s)\right) \mathrm{d} s,
\end{aligned}
$$

and $u_{n}(0)=g\left(u_{n}(T)\right), n=0,1,2, \ldots$

Let $y_{0}=u_{0}, n=1,2,3, \ldots$. By Lemma 10, the Cauchy problem

$$
\begin{gathered}
{ }^{C} D_{0+}^{\delta} y_{n}(t)-M y_{n}(t)=f\left(t, y_{n-1}(t)\right), \\
y_{n}^{\prime}(0)=0, \quad y_{n}(0)=g\left(y_{n-1}(T)\right),
\end{gathered}
$$

has a unique solution

$$
\begin{aligned}
y_{n}(t)= & g\left(y_{n-1}(T)\right) E_{\delta}\left(M t^{\delta}\right) \\
& +\int_{0}^{t}(t-s)^{\delta-1} E_{\delta, \delta}\left(M(t-s)^{\delta}\right) f\left(s, y_{n-1}(s)\right) \mathrm{d} s,
\end{aligned}
$$

which is (69).

In the following, we prove $y_{n}$ converging uniformly to $u^{*}$ on $J$.

For $t \in J$, we have

$$
\begin{aligned}
\mid y_{n}(t) & -u_{n}(t) \mid \\
\leq & E_{\delta}\left(M T^{\delta}\right)\left|g\left(y_{n-1}(T)\right)-g\left(u_{n}(T)\right)\right| \\
& +\int_{0}^{T}(T-s)^{\delta-1} E_{\delta, \delta}\left(M T^{\delta}\right) \\
& \times\left|f\left(s, y_{n-1}(s)\right)-f\left(s, u_{n-1}(s)\right)\right| \mathrm{d} s \\
\leq & E_{\delta}\left(M T^{\delta}\right) g^{\prime}\left(\xi_{n}\right)\left|y_{n-1}(T)-u_{n}(T)\right| \\
\leq & b E_{\delta}\left(M T^{\delta}\right)\left(\left\|u_{n}-u_{n-1}\right\|+\left\|y_{n-1}-u_{n-1}\right\|\right) \\
& +\frac{\kappa T^{\delta} E_{\delta, \delta}\left(M T^{\delta}\right)}{\delta}\left\|y_{n-1}-u_{n-1}\right\| \\
\leq & b E_{\delta}\left(M T^{\delta}\right)\left\|u_{n}-u_{n-1}\right\| \\
+ & \left(b E_{\delta}\left(M T^{\delta}\right)+\frac{\kappa T^{\delta} E_{\delta, \delta}\left(M T^{\delta}\right)}{\delta}\right)\left\|y_{n-1}-u_{n-1}\right\|
\end{aligned}
$$

where $\xi_{n}$ is between $u_{n}(T)$ and $y_{n-1}(T)$.

Therefore,

$$
\left\|y_{n}-u_{n}\right\| \leq b E_{\delta}\left(M T^{\delta}\right)\left\|u_{n}-u_{n-1}\right\|+\varrho_{0}\left\|y_{n-1}-u_{n-1}\right\| .
$$
show

Because $0<\kappa<\delta\left(1-b E_{\delta}\left(M T^{\delta}\right)\right) / T^{\delta} E_{\delta, \delta}\left(M T^{\delta}\right)$, we can

$$
0<\varrho_{0}=b E_{\delta}\left(M T^{\delta}\right)+\frac{\kappa T^{\delta} E_{\delta, \delta}\left(M T^{\delta}\right)}{\delta}<1 .
$$


On the other hand, by (73), we have

$$
\begin{aligned}
& \left\|u_{n}-u_{n-1}\right\| \\
& =\max _{t \in J} \mid\left(u_{n}(0)-u_{n-1}(0)\right) E_{\delta}\left(M t^{\delta}\right) \\
& \quad+\int_{0}^{t}(t-s)^{\delta-1} E_{\delta, \delta}\left(M(t-s)^{\delta}\right) \\
& \quad \times\left(f\left(s, u_{n-1}(s)\right)-f\left(s, u_{n-2}(s)\right)\right) \mathrm{d} s \mid \\
& \leq E_{\delta}\left(M T^{\delta}\right) g^{\prime}\left(\eta_{n}\right)\left\|u_{n}-u_{n-1}\right\| \\
& +\int_{0}^{T}(T-s)^{\delta-1} E_{\delta, \delta}\left(M T^{\delta}\right) \\
& \times\left|f\left(s, u_{n-1}(s)\right)-f\left(s, u_{n-2}(s)\right)\right| \mathrm{d} s \\
& \leq b E_{\delta}\left(M T^{\delta}\right)\left\|u_{n}-u_{n-1}\right\| \\
& +\frac{\kappa T^{\delta} E_{\delta, \delta}\left(M T^{\delta}\right)}{\delta}\left\|u_{n-1}-u_{n-2}\right\|,
\end{aligned}
$$

It follows that $0<\kappa<\delta\left(1-b E_{\delta}\left(M T^{\delta}\right)\right) / T^{\delta} E_{\delta, \delta}\left(M T^{\delta}\right)$ from $0<\varrho_{1}<1$.

By (80), we can easily get

$$
\begin{aligned}
\left\|u_{n}-u_{n-1}\right\| & \leq \varrho_{1}\left\|u_{n-1}-u_{n-2}\right\| \\
& \leq \varrho_{1}^{2}\left\|u_{n-2}-u_{n-2}\right\| \leq \cdots \leq \varrho_{1}^{n-1}\left\|u_{1}-u_{0}\right\| .
\end{aligned}
$$

Substituting (81) into (77), we have

$$
\begin{aligned}
& \left\|y_{n}-u_{n}\right\| \\
& \quad \leq b E_{\delta}\left(M T^{\delta}\right) \varrho_{1}^{n-1}\left\|u_{1}-u_{0}\right\|+\varrho_{0}\left\|y_{n-1}-u_{n-1}\right\| .
\end{aligned}
$$

It is easy to show that

$$
\begin{aligned}
\left\|y_{n}-u_{n}\right\| \leq & b E_{\delta}\left(M T^{\delta}\right)\left(\varrho_{1}^{n-1}+\varrho_{0} \varrho_{1}^{n-2}\right)\left\|u_{1}-u_{0}\right\| \\
& +\varrho_{0}^{2}\left\|y_{n-2}-u_{n-2}\right\| \\
\leq & b E_{\delta}\left(M T^{\delta}\right)\left(\varrho_{1}^{n-1}+\varrho_{0} \varrho_{1}^{n-2}+\varrho_{0}^{2} \varrho_{1}^{n-3}\right)\left\|u_{1}-u_{0}\right\| \\
& +\varrho_{0}^{3}\left\|y_{n-3}-u_{n-3}\right\| \\
\leq & \ldots \\
\leq & b E_{\delta}\left(M T^{\delta}\right)\left(\varrho_{1}^{n-1}+\varrho_{0} \varrho_{1}^{n-2}+\varrho_{0}^{2} \varrho_{1}^{n-3}+\cdots+\varrho_{0}^{n-1}\right) \\
& \times\left\|u_{1}-u_{0}\right\|+\varrho_{0}^{n}\left\|y_{0}-u_{0}\right\| \\
= & b E_{\delta}\left(M T^{\delta}\right)\left(\varrho_{1}^{n-1}+\varrho_{0} \varrho_{1}^{n-2}+\varrho_{0}^{2} \varrho_{1}^{n-3}+\cdots+\varrho_{0}^{n-1}\right) \\
& \times\left\|u_{1}-u_{0}\right\| .
\end{aligned}
$$

We claim $\varrho_{0}>\varrho_{1}$ from (71).

If $\varrho_{0} \leq \varrho_{1}$, we have

$$
b E_{\delta}\left(M T^{\delta}\right)+\frac{\kappa T^{\delta} E_{\delta, \delta}\left(M T^{\delta}\right)}{\delta} \leq \frac{\kappa T^{\delta} E_{\delta, \delta}\left(M T^{\delta}\right)}{\delta\left(1-b E_{\delta}\left(M T^{\delta}\right)\right)} .
$$

So

$$
\kappa \geq \frac{\delta\left(1-b E_{\delta}\left(M T^{\delta}\right)\right)}{T^{\delta} E_{\delta, \delta}\left(M T^{\delta}\right)},
$$

which is a contradiction to the hypothesis of the theorem.

Therefore, it follows from (78) that

$$
\begin{aligned}
& \left\|y_{n}-u_{n}\right\| \\
& \quad \leq \frac{b E_{\delta}\left(M T^{\delta}\right)\left(\varrho_{0}^{n}-\varrho_{1}^{n}\right)}{\varrho_{0}-\varrho_{1}}\left\|u_{1}-u_{0}\right\| \rightarrow 0, \quad \text { as } n \rightarrow \infty .
\end{aligned}
$$

By (72) and (86), we can show that

$$
\left\|y_{n}-u^{*}\right\| \leq\left\|y_{n}-u_{n}\right\|+\left\|u_{n}-u^{*}\right\| \longrightarrow 0, \quad \text { as } n \longrightarrow \infty .
$$

Then for any initial value $u_{0} \in\left[\beta_{0}, \alpha_{0}\right]$, the sequence $\left\{y_{n}\right\}$ is defined by (69) converging uniformly to the unique solution $u^{*}$ of the boundary value problem (1) on the $\left[\beta_{0}, \alpha_{0}\right]$.

In view of (61), we have

$$
\begin{aligned}
\left\|u_{n}-u^{*}\right\| & \leq\left(\frac{\kappa T^{\delta} E_{\delta, \delta}\left(M T^{\delta}\right)}{\delta\left(1-a E_{\delta}\left(M T^{\delta}\right)\right)}\right)^{n}\left\|\alpha_{0}-\beta_{0}\right\| \\
& =\varrho_{1}^{n}\left\|\alpha_{0}-\beta_{0}\right\| .
\end{aligned}
$$

According to (86), we can show

$$
\left\|y_{n}-u_{n}\right\| \leq \frac{b E_{\delta}\left(M T^{\delta}\right)\left(\varrho_{0}^{n}-\varrho_{1}^{n}\right)}{\varrho_{0}-\varrho_{1}}\left\|\alpha_{0}-\beta_{0}\right\| .
$$


It is easy to see that

$$
\varrho_{0}-\varrho_{1}=b E_{\delta}\left(M T^{\delta}\right)\left(1-\varrho_{1}\right) .
$$

By (88) and (89), we can obtain

$$
\begin{aligned}
\left\|y_{n}-u^{*}\right\| \leq & \varrho_{1}^{n}\left\|\alpha_{0}-\beta_{0}\right\| \\
& +\frac{b E_{\delta}\left(M T^{\delta}\right)\left(\varrho_{0}^{n}-\varrho_{1}^{n}\right)}{\varrho_{0}-\varrho_{1}}\left\|\alpha_{0}-\beta_{0}\right\| \\
= & \left(\varrho_{1}^{n}+\frac{\varrho_{0}^{n}-\varrho_{1}^{n}}{1-\varrho_{1}}\right)\left\|\alpha_{0}-\beta_{0}\right\| \\
= & \frac{\varrho_{0}^{n}-\varrho_{1}^{n+1}}{1-\varrho_{1}}\left\|\alpha_{0}-\beta_{0}\right\| .
\end{aligned}
$$

Hence, the error estimate of $\left\{y_{n}\right\}$ is

$$
\left\|y_{n}-u^{*}\right\| \leq \frac{\varrho_{0}^{n}-\varrho_{1}^{n+1}}{1-\varrho_{1}}\left\|\alpha_{0}-\beta_{0}\right\| .
$$

\section{Example}

We consider the boundary value problem

$$
\begin{gathered}
{ }^{C} D_{0+}^{3 / 2} u(t)-\frac{1}{4} u(t)=\frac{t}{10} \arctan u(t)+\frac{t^{2}}{10}, \\
u^{\prime}(0)=0, \quad u(0)=\frac{1}{5} \arctan u(1) .
\end{gathered}
$$

Let $f(t, u)=(t / 10) \arctan u+t^{2} / 10$ and $g(u)=$ $(1 / 5) \arctan u$.

It is obvious that $M=1 / 4, T=1, \delta=3 / 2$, and $b=$ $\sup _{u \in \mathbb{R}} g^{\prime}(u)=1 / 5<1 / E_{\delta}\left(M T^{\delta}\right)(\approx 0.834179)$. Then $(\mathrm{H} 1)$ holds.

We take $\beta(t)=0$ for $t \in J$.

Hence, $\beta(0)=0, g(\beta(1))=0$, and $b_{\beta}(t)=0 .{ }^{C} D_{0+}^{\delta} \beta(t)=$ $0, f(t, \beta(t))=0$, and then $\beta(t)$ is an upper solution of the boundary value problem (93).

Let $\alpha(t)=5 t^{3 / 2} / 6 \sqrt{\pi}+5 t / 8 \sqrt{\pi}+35 / 48 \sqrt{\pi} \leq 35 / 16 \sqrt{\pi}$ for $t \in J$.

We have $\alpha^{\prime}(0)=5 / 8 \sqrt{\pi}, \alpha(0)=35 / 48 \sqrt{\pi} \approx 0.411388$, $g(\alpha(1))=(1 / 5) \arctan (35 / 16 \sqrt{\pi}) \approx 0.177966$, and $a_{\alpha}(t)=0$. Consider

$$
\begin{aligned}
& { }^{C} D_{0+}^{\delta} \alpha(t)-\frac{1}{4} \alpha(t) \\
& \geq \frac{5}{8}-\frac{35}{4 \times 16 \sqrt{\pi}} \approx 0.316459 \\
& >0.188983 \approx f\left(1, \frac{35}{16 \sqrt{\pi}}\right) \\
& \quad \geq f(t, \alpha(t))
\end{aligned}
$$

for $t \in J$. Then $\alpha(t)$ is a lower solution of the boundary value problem (93). And $\beta(t) \leq \alpha(t)$.
It is easy to verify that the (H2) holds. By using Theorem 17, we know the problem (93) has minimal and maximal solutions in $[\beta, \alpha]$.

Let $\kappa=1 / 10$; we know $\kappa<\delta\left(1-b E_{\delta}\left(M T^{\delta}\right)\right) /$ $T^{\delta} E_{\delta, \delta}\left(M T^{\delta}\right) \approx 0.905853$.

By calculating, we have

$$
\varrho_{0} \approx 0.323682, \quad \varrho_{1} \approx 0.110393 .
$$

It is obvious that

$$
\begin{array}{r}
0 \leq f\left(t, x_{2}\right)-f\left(t, x_{1}\right) \leq \frac{1}{10}\left(x_{2}-x_{1}\right)=\kappa\left(x_{2}-x_{1}\right), \\
\text { for any } x_{1} \leq x_{2}, \quad t \in J .
\end{array}
$$

By Theorem 19, we can get the problem (93) has a unique solution on the $\left[\beta_{0}, \alpha_{0}\right]$.

For any initial value $u_{0} \in\left[\beta_{0}, \alpha_{0}\right]$, the sequence $\left\{y_{n}\right\}$ defined by

$$
\begin{aligned}
y_{n}(t)= & \frac{1}{5} \arctan \left(y_{n-1}(1)\right) E_{3 / 2}\left(\frac{1}{4} t^{3 / 2}\right) \\
& +\int_{0}^{t}(t-s)^{1 / 2} E_{3 / 2,3 / 2}\left(\frac{1}{4}(t-s)^{3 / 2}\right) \\
& \times\left(\frac{s}{10} \arctan y_{n-1}(s)+\frac{s^{2}}{10}\right) \mathrm{d} s,
\end{aligned}
$$

converging uniformly to the unique solution $u^{*}$ of the boundary value problem (93) on the $\left[\beta_{0}, \alpha_{0}\right]$, and its error estimate is

$$
\left\|y_{n}-u^{*}\right\| \leq \frac{\varrho_{0}^{n}-\varrho_{1}^{n+1}}{1-\varrho_{1}}\left\|\alpha_{0}-\beta_{0}\right\|=\frac{35\left(\varrho_{0}^{n}-\varrho_{1}^{n+1}\right)}{16 \sqrt{\pi}\left(1-\varrho_{1}\right)},
$$

where $y_{0}=u_{0}$.

We take $u_{0}=0$. For $n=3$, its error is not more than 0.046841 ; for $n=5$, its error is not more than 0.00492662 ; for $n=8$, its error is not more than 0.000167155 ; for $n=10$, its error is not more than 0.0000175132 .

\section{Conflict of Interests}

The authors declare that there is no conflict of interests regarding the publication of this paper.

\section{Acknowledgments}

This work is supported by Natural Science Foundation of China (no. 11171220) and Support Projects of University of Shanghai for Science and Technology (no. 14XPM01).

\section{References}

[1] K. Diethelm and A. D. Freed, "On the solution of nonlinear fractional order differential equations used in the modeling of 
viscoplasticity," in Science Computing in Chemical EngineeringComputational Fluid Dynamics, Reaction Engineering and Molecular Properties1999, F. Keil, W. Mackens, H. Voss, and J. Werther, Eds., pp. 217-224, Springer, Heidelberg, Germany.

[2] R. Hilfer, Applications of Fractional Calculus in Physics, World Scientific, Singapore.

[3] A. A. Kilbas, H. M. Srivastava, and J. J. Trujillo, Theory and Applications of Fractional Differential Equations, NorthHolland Mathematics Studies, Elsevier Science, Amsterdam, The Netherlands, 2006.

[4] K. Diethelm, The Analysis of Fractional Differential Equation, Springer, Heidelberg, Germany, 2010.

[5] S. Zhang, "Existence of a solution for the fractional differential equation with nonlinear boundary conditions," Computers and Mathematics with Applications, vol. 61, no. 4, pp. 1202-1208, 2011.

[6] R. W. Ibrahim and M. Darus, "Subordination and superordination for univalent solutions for fractional differential equations," Journal of Mathematical Analysis and Applications, vol. 345, no. 2, pp. 871-879, 2008.

[7] S. Ladaci, J. J. Loiseau, and A. Charef, "Fractional order adaptive high-gain controllers for a class of linear systems," Communications in Nonlinear Science and Numerical Simulation, vol. 13, no. 4, pp. 707-714, 2008.

[8] X. Liu, L. Lin, and H. Fang, "Existence of positive solutions for nonlocal boundary value problem of fractional differential equation," Central European Journal of Physics, vol. 11, pp. 14231432, 2013.

[9] M. Jia and X. Liu, "The existence of positive solutions for fractional differential equations with integral and disturbance parameter in boundary conditions," Abstract and Applied Analysis, vol. 2014, Article ID 131548, 14 pages, 2014.

[10] M. Jia and X. Liu, "Multiplicity of solutions for integral boundary value problems of fractional differential equations with upper and lower solutions," Applied Mathematics and Computation, vol. 232, pp. 313-323, 2014.

[11] Y. Liu, "Existence of solutions for impulsive differential models on half lines involving Caputo fractional derivatives," Communications in Nonlinear Science and Numerical Simulation, vol. 18, no. 10, pp. 2604-2625, 2013.

[12] Y. Liu, "Application of Avery-Peterson fixed point theorem to nonlinear boundary value problem of fractional differential equation with the Caputo's derivative," Communications in Nonlinear Science and Numerical Simulation, vol. 17, no. 12, pp. 4576-4584, 2012.

[13] W. Wang, X. Yang, and J. Shen, "Boundary value problems involving upper and lower solutions in reverse order," Journal of Computational and Applied Mathematics, vol. 230, no. 1, pp. $1-7,2009$.

[14] F. Li, M. Jia, X. Liu, C. Li, and G. Li, "Existence and uniqueness of solutions of second-order three-point boundary value problems with upper and lower solutions in the reversed order," Nonlinear Analysis, Theory, Methods and Applications, vol. 68, no. 8, pp. 2381-2388, 2008.

[15] F. Li, J. Sun, and M. Jia, "Monotone iterative method for the second-order three-point boundary value problem with upper and lower solutions in the reversed order," Applied Mathematics and Computation, vol. 217, no. 9, pp. 4840-4847, 2011.

[16] L. Lin, X. Liu, and H. Fang, "Method of upper and lower solutions for fractional differential equations," Electronic Journal of Differential Equations, vol. 100, pp. 1-13, 2012.
[17] G. Wang, R. P. Agarwal, and A. Cabada, "Existence results and the monotone iterative technique for systems of nonlinear fractional differential equations," Applied Mathematics Letters, vol. 25, pp. 1019-1024, 2012.

[18] Z. Liu and J. Sun, "Nonlinear boundary value problems of fractional functional integro-differential equations," Computers \& Mathematics with Applications, vol. 64, pp. 3228-3234, 2012.

[19] D. Guo, J. Sun, and Z. Liu, Functional Method For Nonlinear Ordinary Differential Equation, Shandong Science and Technology Press, Jinan, China, 1995, Chinese. 


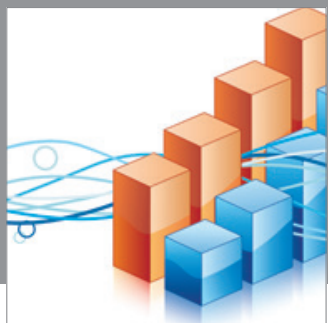

Advances in

Operations Research

mansans

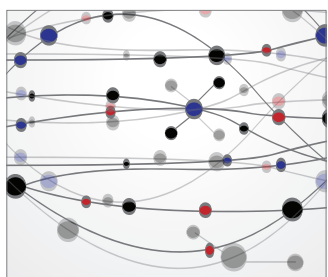

The Scientific World Journal
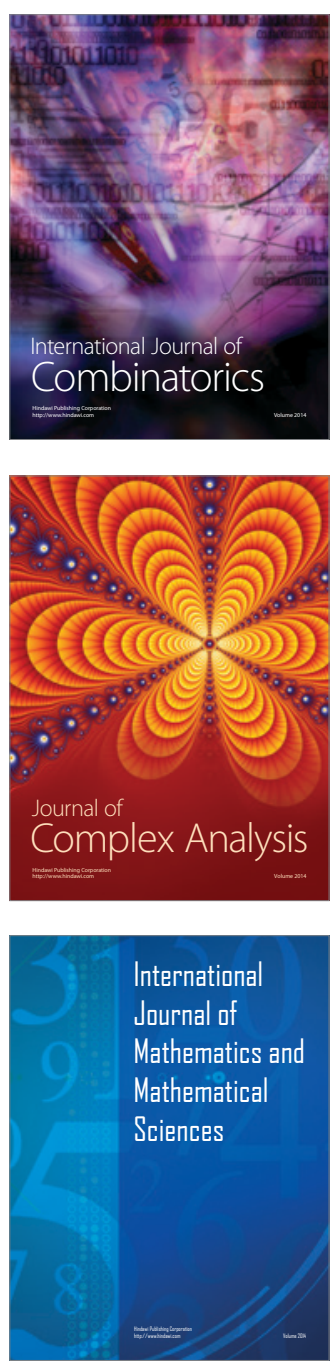
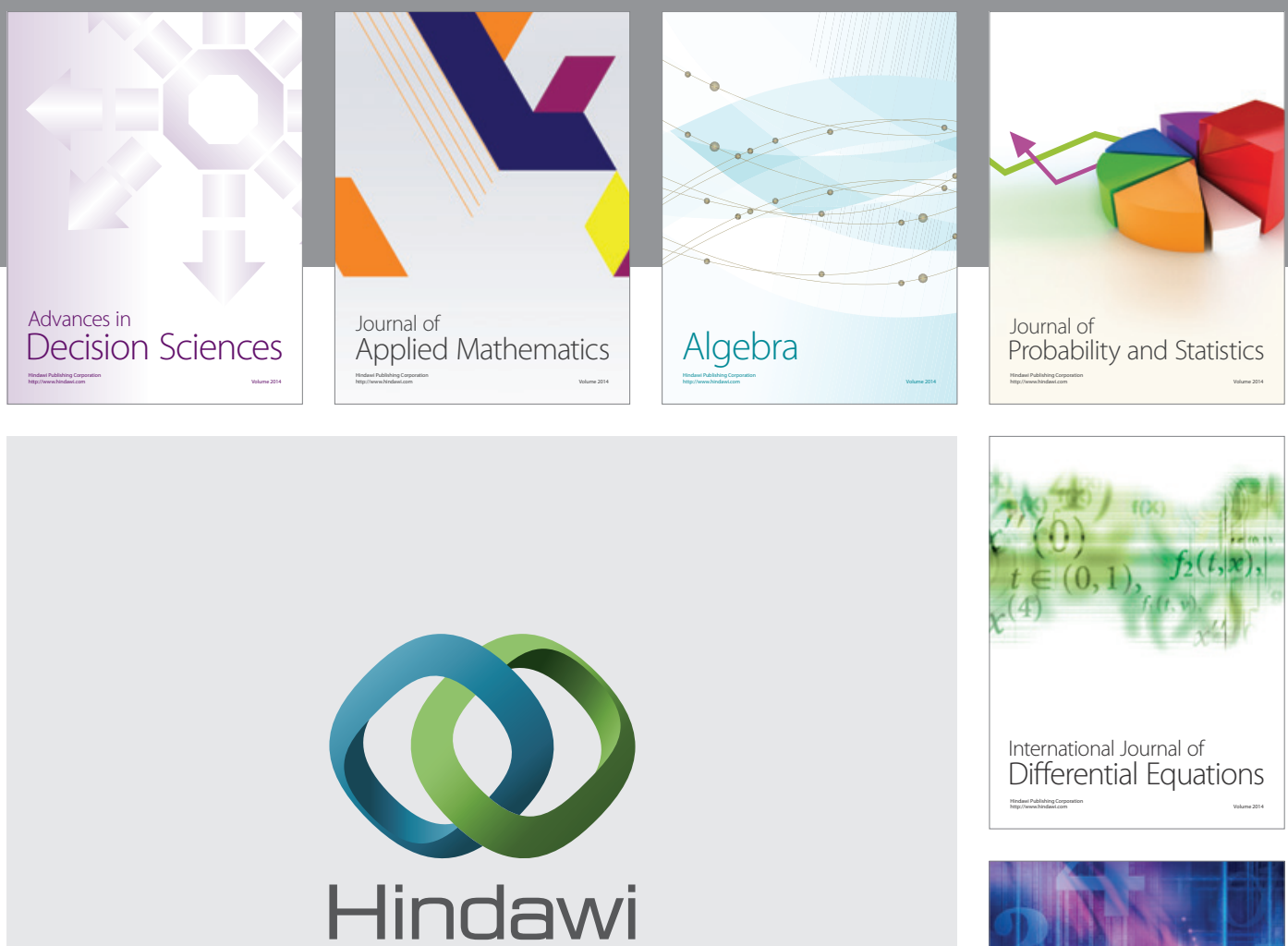

Submit your manuscripts at http://www.hindawi.com
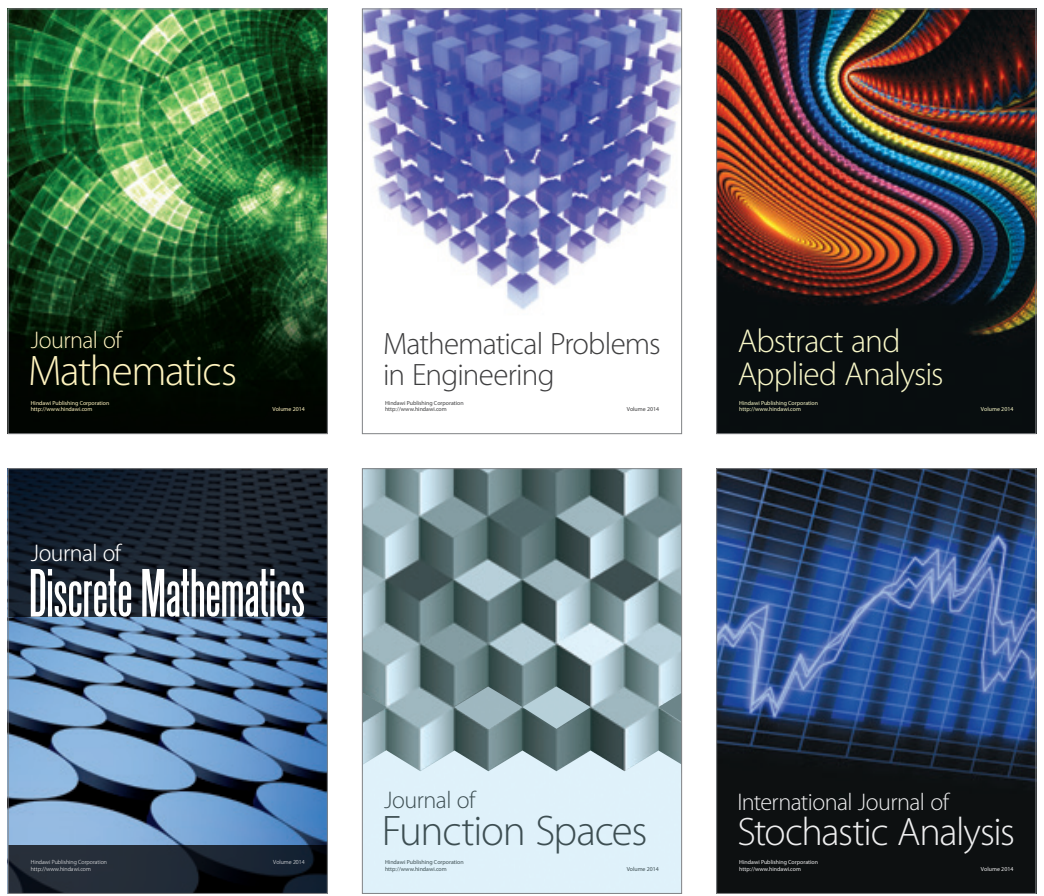

Journal of

Function Spaces

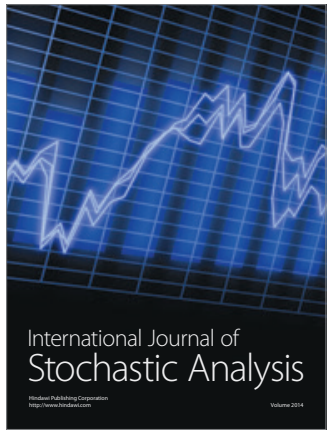

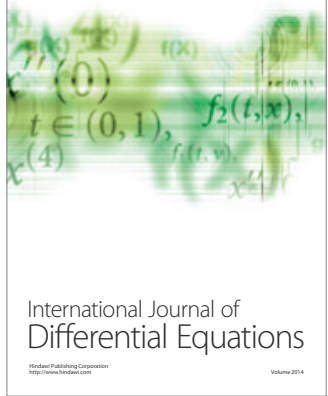
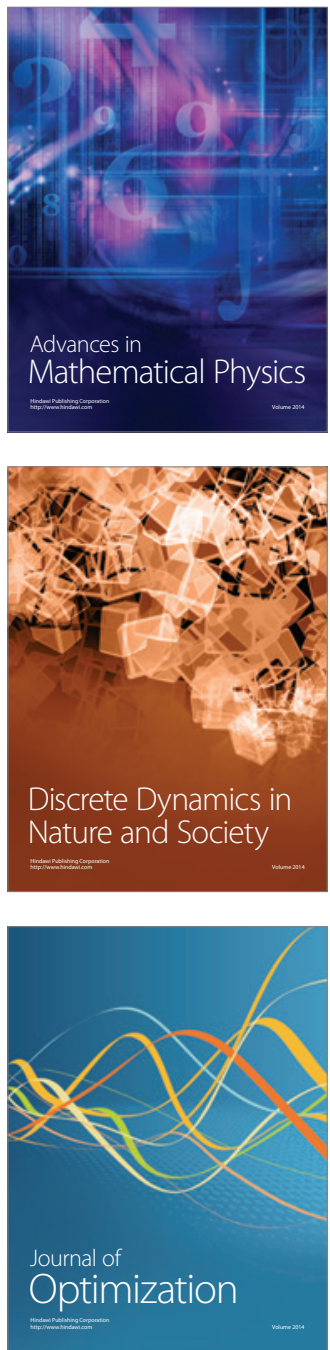